\title{
3D-speckle-tracking echocardiography correlates with CMR diagnosis of acute myocarditis
}

\author{
Philip Goody ${ }^{1}$, Sebastian Zimmer ${ }^{1}$, Angela Zimmer ${ }^{1}$, Jens Kreuz ${ }^{2}$, Marc Becher ${ }^{1}$, \\ Alexander Isaak ${ }^{3}$, Georg Nickenig ${ }^{1}$, Christoph Hammerstingl ${ }^{4}$, and Vedat Tiyerili ${ }^{1}$ \\ ${ }^{1}$ Heart Center Bonn, University Hospital Bonn \\ ${ }^{2}$ Affiliation not available \\ ${ }^{3}$ University Hospital Bonn \\ ${ }^{4}$ Zentrum für Herz- und Gefäßmedizin, Mediapark Köln
}

February 17, 2021

\begin{abstract}
Abstract Background: The diagnostic importance of three-dimensional (3D) speckle-tracking strain-imaging echocardiography in patients with acute myocarditis has not yet been assessed. The aim of this study was to test the sensitivity and specificity of 3D-speckle-tracking echocardiography as compared to CMR (cardiovascular magnetic resonance imaging) for the diagnosis of acute myocarditis. Methods and Results: 45 patients that were admitted to the Medical Clinic II of the University Hospital Bonn with clinically suspected myocarditis were enrolled in our study (71\% male, mean age: $43.9 \pm 16.3$ years, peak troponin level: $1.38 \pm 3.51 \mathrm{ng} / \mathrm{ml}$ ). 3D full-volume echocardiographic images were obtained and offline speckle-tracking analysis of regional and global LV deformation was performed. All patients received CMR scans and myocarditis was diagnosed in 29 subjects. The 16 patients, in whom myocarditis was excluded by CMR, served as controls. Regional changes in myocardial texture and tissue edema (diagnosed by CMR) were significantly associated with regional impairment of circumferential, longitudinal, and radial strain, as well as regional 3D displacement and total 3D strain. The 3D diastolic strain index was not associated with pathological findings in the CMR. However, the 3D global longitudinal strain (GLS) outperformed well-known 2D parameters associated with myocarditis, such as LVEF and LVEDV. Conclusions: This is the first study examining the use of 3D-speckletracking echocardiography in patients with acute myocarditis. Global longitudinal strain was significantly associated with and impaired in patients with myocarditis. Therefore, 3D echocardiography could become a useful diagnostic tool in the primary diagnosis of myocarditis.
\end{abstract}

\section{D-speckle-tracking echocardiography correlates with CMR diagnosis of acute myocarditis}

Short title: 3D-Speckle-tracking in myocarditis

Philip Roger Goody ${ }^{1, \#}$, MD; Sebastian Zimmer ${ }^{1, \#, ~ M D ; ~ A n g e l a ~ Z i m m e r ~}{ }^{1}$; Jens Kreuz ${ }^{1}$, MD; Marc Ulrich Becher $^{1}$, MD; Alexander Isaak ${ }^{2}$, MD; Georg Nickenig ${ }^{1}$, MD; Christoph Hammerstingl ${ }^{1}$, MD; and Vedat Tiyerili ${ }^{1}, \mathrm{MD}$

${ }^{1}$ Heart Center Bonn, Department of Medicine II

University Hospital Bonn, Venusberg-Campus 1, 53127 Bonn, Germany

${ }^{2}$ Department of Radiology

University Hospital Bonn, Venusberg-Campus 1, 53125 Bonn, Germany.

\# contributed equally 
Word count: 2626 (including abstract); Figures: 4; Tables: 2; References: 29

Key words: 3D-speckle-tracking; Myocarditis; Cardiovascular magnetic resonance imaging

Corresponding author: Vedat Tiyerili, MD

Medizinische Klinik und Poliklinik II, Universitätsklinikum Bonn

Venusberg-Campus 1, 53125 Bonn, Germany

e-mail: Vedat.Tiyerili@ukb.uni-bonn.de

phone: 0049/228-28715217, fax: 0049/228-28711271

\section{Acknowledgements}

PRG is funded by the Else-Kröner-Fresenius foundation. VT, SZ and GN are supported by the Deutsche Forschungsgemeinschaft (SFB TRR 259/1). We thank Dr. Meghan Campbell for critical reading of the manuscript.

\section{Conflict of interest statement}

There are no conflicts of interest to disclose

\section{Highlights}

- Regional changes in myocardial texture and tissue edema (diagnosed by CMR) were significantly associated with regional impairment of circumferential, longitudinal, and radial strain, as well as regional 3D displacement and total 3D strain.

- 3D global longitudinal strain (GLS) outperformed well-known 2D parameters associated with myocarditis, such as LVEF and LVEDV

- 3D-speckle-tracking echocardiography could become a useful diagnostic tool in the diagnosis of myocarditis

\section{Abstract}

Background: The diagnostic importance of three-dimensional (3D) speckle-tracking strain-imaging echocardiography in patients with acute myocarditis has not yet been assessed. The aim of this study was to test the sensitivity and specificity of 3D-speckle-tracking echocardiography as compared to CMR (cardiovascular magnetic resonance imaging) for the diagnosis of acute myocarditis.

Methods and Results: 45 patients that were admitted to the Medical Clinic II of the University Hospital Bonn with clinically suspected myocarditis were enrolled in our study ( $71 \%$ male, mean age: $43.9 \pm 16.3$ years, peak troponin level: $1.38 \pm 3.51 \mathrm{ng} / \mathrm{ml}$ ). 3D full-volume echocardiographic images were obtained and offline speckle-tracking analysis of regional and global LV deformation was performed. All patients received CMR scans and myocarditis was diagnosed in 29 subjects. The 16 patients, in whom myocarditis was excluded by CMR, served as controls. Regional changes in myocardial texture and tissue edema (diagnosed by CMR) were significantly associated with regional impairment of circumferential, longitudinal, and radial strain, as well as regional 3D displacement and total 3D strain. The 3D diastolic strain index was not associated with pathological findings in the CMR. However, the 3D global longitudinal strain (GLS) outperformed well-known 2D parameters associated with myocarditis, such as LVEF and LVEDV.

Conclusions: This is the first study examining the use of 3D-speckle-tracking echocardiography in patients with acute myocarditis. Global longitudinal strain was significantly associated with and impaired in patients with myocarditis. Therefore, 3D echocardiography could become a useful diagnostic tool in the primary diagnosis of myocarditis.

\section{Introduction:}


Myocarditis is an inflammatory disease of the heart muscle frequently resulting from viral infection and/or post viral immune-mediated responses. Its clinical manifestation can range from essentially asymptomatic presentations showing symptoms of myocardial infarction to severe illness, including cardiogenic shock due to chronic or acute heart failure (HF) (1). Due to this broad variety of presentations, the diagnosis of myocarditis cannot usually be made based on clinical findings. Myocarditis can result in dilated cardiomyopathy (DCM) and is currently the leading cause for heart transplantation.

The diagnostic accuracy of clinical history, physical examination, electrocardiography, and serology are generally not adequate for an accurate diagnosis of myocarditis. Furthermore, the findings during acute myocarditis in conventional echocardiography are not specific (2). Endomyocardial biopsy, with further immunohistochemical and biochemical workup, remains the most widely accepted standard technique, however, it may not be appropriate for many patients, especially those with less severe disease. For the noninvasive imaging techniques, cardiac magnetic resonance imaging (CMR) has become the most important diagnostic tool for myocarditis (3).

Consensus CMR criteria for myocarditis (including visualization of edema with cellular swelling and using early and late enhancement methods) has standardized CMR protocols for assessing myocarditis $(3,4)$. Echocardiography is currently recommended in the initial diagnostic evaluation of all patients with suspected myocarditis (5). Echocardiography in myocarditis is typically performed to visualize the associated functional abnormalities, such as wall thickness and pericardial effusion. However, many patients with less severe cases of myocarditis present with a normal echocardiogram or unspecific echocardiographic findings, which diminishes the diagnostic value of this technique, owing to poor specificity and high variability (2).

Two-dimensional speckle-tracking echocardiography can quantitatively measure myocardial mechanics (strain and strain rate) in longitudinal, radial, and circumferential directions (6-8). In many clinical settings, such as cardiomyopathy or coronary artery disease, a decrease in the longitudinal strain shown by two-dimensional speckle-tracking echocardiography has been found to indicate left-ventricular dysfunction before the occurrence of changes in left-ventricular ejection fraction $(9,10)$. Hsiao et al. demonstrated in acute myocarditis that left-ventricular strain and strain rate may be promising diagnostic and prognostic tools, even in patients with preserved left-ventricular ejection fraction (11). However, the diagnostic importance of a novel technique, three-dimensional (3D) speckle-tracking strain imaging echocardiography, in patients with acute myocarditis has not yet been assessed. The aim of this study was to test the sensitivity and specificity of 3D-speckle-tracking echocardiography versus CMR in the diagnosis of acute myocarditis.

\section{Methods}

We analyzed acquired data sets of 45 patients with suspected acute myocarditis in this observational study. Inclusion criteria were (a) clinical symptoms compatible with a diagnosis of acute myocarditis, such as fever, viral prodromes, chest pain, palpitations, or dyspnea within six weeks of admission, (b) evidence of structural or functional abnormalities by echocardiography or evidence of myocardial damage as indicated by an elevation of at least one biomarker (troponin I level $>0.1 \mathrm{ng} / \mathrm{ml}$ or creatine kinase MB fraction 0.5-3.6 $\mu \mathrm{g} / \mathrm{l}$ ), and (c) absence of evidence of coronary artery disease by coronary angiography. The exclusion criteria were previous heart surgery, known coronary artery disease (CAD), severe valvular disease, and a poor echocardiographic window. Controls were individuals with no history of cardiovascular disease who had normal echocardiography and no signs of acute myocarditis via CMR.

\subsection{Two-dimensional transthoracic echocardiography}

Each patient underwent standardized two-dimensional (2D) transthoracic echocardiography for the determination of left- and right-ventricular functional parameters and dimensions, according to the recommendations of the American Society of Echocardiography, using a commercially available ultrasound scanner (Vivid 7, General Electric Medical Health, Waukesha, Wisconsin, USA) with a $2.5 \mathrm{MHz}$ phased-array transducer (12, 13). Echocardiographic views, including apical four- and two-chamber views (4CV, $2 \mathrm{CV}$ ) and parasternal (long- and short-axis views), with the patient in the left lateral decubitus position, were obtained in twodimensional, three-dimensional, and color tissue Doppler imaging (TDI) modes. The 2D ejection fraction 
(EF) was calculated by using Simpson's rule from the $4 \mathrm{CV}$ image. The E/e' ratio was determined by measuring the TDI-derived systolic and diastolic velocities of the septal mitral valve annulus, according to the recommendations of the American Society of Echocardiography.

\subsection{Three-dimensional speckle-tracking analysis}

A 3D full-volume data set of the ventricle was obtained with gated (five beats) acquisition and stored on an echocardiographic imaging server (XCELERA, Philips Medical Systems, Koninklijke N.V.). Offline 3D ST analyses of the gray-scale images obtained by 3D echocardiography were performed with commerciallyavailable software (TomTec Imaging Systems GmbH, Unterschleissheim, Germany). Sector size and depth were optimized to obtain the highest possible volume rates, reaching 17 to 20 frames/sec in the contrast 3D full-volume mode. The best real-time 3D echocardiography (3DE) dataset was selected and analyzed offline by an experienced investigator who was blinded to the results of the CMR and 2DE measurements.

\subsection{Cardiovascular magnetic resonance imaging}

All patients received CMR-scans within $72 \mathrm{~h}$ of admission in accordance to our site-specific SOP. All examinations were performed on a 1.5 Tesla system (Intera 1.5 T; Philips Healthcare, Best, The Netherlands). A 32-channel torso coil with digital interface was used for signal reception. ECG-gated steady-state free precession cine images were obtained, including short axis (SA), vertical long axis (VLA), horizontal long axis (HLA), and left-ventricular outflow track (LVOT) stacks. The left ventricle was fully covered in the SA stack. Edema-sensitive black blood T2-weighted short-tau inversion recovery (STIR) sequences were performed in SA and transverse orientations. Early gadolinium enhancement was assessed using transverse free-breathing fast-spin echo T1-weighted images, which were acquired in three identical slices taken both before and after intravenous injection of a double-dose bolus of $0.2 \mathrm{mmol} / \mathrm{kg}$ of body weight of gadobutrol (Gadovist, Bayer Healthcare, Leverkusen, Germany) using the body coil for signal reception. Late gadolinium enhancement (LGE) imaging was achieved by segmented inversion recovery gradient-echo sequences in SA, VLA, and HLA orientations. The optimal inversion time was determined by using the Look-Locker technique.

\subsection{Statistical Analysis}

We used the Kolmogorov-Smirnov test to examine the normal distribution of continuous variables. Continuous data were expressed as mean values \pm standard deviation. Two-tailed $p$-values were calculated and were considered to be significant if they ranged below 0.05. For comparison between two groups, we used the Student t test for paired samples. To evaluate the results, in particular their predictive diagnostic value, and to enable a comparison between the results obtained from the cardiac MRI with the results from the speckle-tracking analysis and the control group, we used the following parameters and methods:

To analyze the collected data and its predictive value, we used receiver operating characteristic (ROC) curves and the Youden index $(\mathrm{J})$. The resulting values ranged between 0 and 1 , with values near 0 indicating only low diagnostic value and values near 1 showing very good diagnostic value. For ROC curves, the corresponding areas under the curve (AUC) and their $95 \%$ confidence intervals (CIs) were calculated.

\section{Results}

The study design is depicted in Figure 1. A total of 45 patients with clinically suspected myocarditis who met the inclusion criteria were enrolled in the study. Initially, conventional two-dimensional echocardiography and 3D full-volume echocardiographic images were obtained with offline speckle-tracking analysis of regional and global LV deformation. All patients were subjected to CMR scans, and myocarditis was diagnosed in 29 of the subjects (age $47.7 \pm 17$, male $72 \%$ ). There were 16 patients in whom myocarditis was excluded by CMR, and these patients then served as controls for the study (age $40.4 \pm 15.6$, male $63 \%$ ). The initial clinical characteristics of the patients are shown in Table 1. Body weight, blood pressure, and smoking rates were similar in both groups. The peak troponin and peak CK-MB levels were elevated in patients with myocarditis without reaching significance (peak troponin $2.15 \pm 5.44 \mathrm{ng} / \mathrm{ml}$ vs $0.61 \pm 1.58 \mathrm{ng} / \mathrm{ml}(\mathrm{p}=0.28$ ), CK-MB $20.6 \pm 51.3 \mu \mathrm{g} / \mathrm{l}$ vs $20.4 \pm 63.6 \mu \mathrm{g} / \mathrm{l}(\mathrm{p}=0.17)$ ). The inflammation status of the patients was detected by measuring the peak leukocytes and peak hsCRP levels and did not differ between the two groups. 
The two-dimensional and 3D-echocardiograms were performed within 24 hours after admission to our clinic. The main conventional echocardiographic findings are shown in Table 2. In patients with myocarditis LVEF was significantly reduced $(44.4 \pm 3.2 \%$ vs. $54.3 \pm 4.2(\mathrm{p}=0.03))$. In addition, this was associated with significantly higher LVEDP $(159.3 \pm 14.3 \mathrm{mmHg}$ vs. $118.8 \pm 14.4 \mathrm{mmHg}(\mathrm{p}=0.02))$, higher E/A ratios $(1.9 \pm 0.22$ vs. $1.05 \pm 0.09(\mathrm{p}=0.03))$, and elevated septal $\mathrm{E} / \mathrm{e}$ ' ratios $(12.76 \pm 1.9$ vs. $4.9 \pm 0.67(\mathrm{p}=0.004)$. There was no difference in IVSd, systolic PAP, or TAPSE measurements between the two groups. Furthermore, no difference was detected in the primary ECG findings (Table 2).

Regional changes in myocardial texture and tissue edema, as diagnosed by CMR, were significantly associated with regional impairment of circumferential, longitudinal, and radial strain as well as regional 3D displacement and total 3D strain (Figure 2). The 3D diastolic strain index was not associated with pathological findings in the CMR (Figure 2). 3D global longitudinal strain (GLS) out-performed the well-known 2D parameters associated with myocarditis, such as LVEF and LVEDV (Figure 3). The predictive value of 3D-GLS was confirmed by ROC curve analysis, which demonstrated an area under the curve (AUC) of 0.71 ( $95 \%$ confidence interval 0.53 to $0.83, \mathrm{p}=0.019$, Figure 3 ). Global torsion and twist were not associated with myocarditis in our collective (Figure 3 ).

\section{Discussion}

3D echocardiography has been widely used in clinical settings since its introduction in commercially available 3D-speckle tracking systems. 3D-speckle-tracking has not been regarded as a routine tool for analysis of cardiac function, despite its various advantages compared to two-dimensional (2D) imaging (16). 3D-speckletracking is a novel echocardiographic technique that allows digital tracking and quantification of myocardial deformation as a function of time. The deformation can be assessed as three orthogonal strain values: radial strain (RS), longitudinal strain (LS), and circumferential strain (CS). Strain and strain-rate imaging can assess both circumferential deformation (a negative value in systole, as the circumference of the LV decreases) and radial deformation (a positive value in systole, as the LV wall thickness increases) (17).

Acute myocarditis is characterized by inflammatory myocardial damage that can result in severe leftventricular wall motion abnormalities (1). However, the myocardial damage is mainly circumscribed to the subepicardial layers, whether or not it is associated with any wall motion abnormality. Previous echocardiographical studies of patients with myocarditis have shown a variety of findings (18).

In addition to systolic function, regional wall motion abnormalities, diastolic dysfunction, and changes in echocardiographic image texture have been reported $(2,6,19-21)$. In this study, routine parameters such LVEDP and septal E/e' ratio are significantly elevated due to acute inflammatory left-ventricular damage. Furthermore, LVEF was significantly reduced in patients with acute myocarditis.

The most important result of this study is that 3D global longitudinal strain (GLS) outperformed the wellknown 2D parameters associated with myocarditis such as LVEF and LVEDV (2). A GLS assessment using automated speckle-tracking echocardiography is a technique for detecting and quantifying subtle disturbances in the LV systolic function (22). GLS reflects the longitudinal contraction of the myocardium, and its accuracy has been validated against tagged CMR (23). In the general population and patients with heart failure, GLS was shown to be a superior predictor of cardiac events and all-cause mortality compared to LVEF $(24,25)$. Furthermore, it has also been shown that GLS is a robust prognostic marker following myocardial infarction as well as in patients with cardiomyopathy and aortic stenosis (26-28).

The second relevant finding is that regional changes in myocardial texture and tissue edema, as diagnosed by CMR, were significantly associated with regional impairment of circumferential, longitudinal, and radial strain as well as regional 3D displacement and total 3D strain. Our results also showed that radial strain had the highest accuracy in detecting the segmental myocardial defects that are induced by acute myocarditis. Radial strain represents radially-directed myocardial deformation toward the center of the LV cavity, and thus indicates the degree of LV thickening and thinning motions during the cardiac cycle. Radial strain values are obtained by 3D-speckle-tracking echocardiographic analysis of both basal and apical LV short-axis views. 
Routinely, echocardiography is the first imaging technique used in a clinical setting. Especially in patients with angina and normal LV size and function, the findings are mostly normal, which leads to poor diagnostic accuracy. The gold standard for noninvasive diagnosis of myocarditis is cardiac magnetic resonance imaging (CMR). CMR or invasive diagnostics, such as myocardial biopsy, are often not feasible in the setting of an acute presentation. It has been shown that $2 \mathrm{D}$ echocardiography with strain imaging can help with differential diagnosis and lead to an optimal treatment path for the patient $(10,29)$.

A representative case (shown in Figure 4) demonstrates that decreased myocardial 3D radial strain, as assessed by 3D-speckle tracking, is associated with the CMR findings. However, the major pitfalls of 3Dspeckle-tracking are its dependency on image quality and its ability to define the endocardial and epicardial boundaries by random noise and relatively low temporal resolution.

2D echocardiography has generally played only a minor role in confirming the diagnosis of acute myocarditis. The underlying reasons are the apparently normal findings that it gives and a lack of specific features for less severe forms of myocarditis.

The advent of novel echocardiographic modalities, such as strain echocardiography, has dramatically expanded the scope of echocardiography, which can provide an accurate bedside assessment of regional contractility and can identify global longitudinal myocardial dysfunction derived from edema in acute myocarditis. Interestingly, a decrease in myocardial GLS, as assessed by the 3D-speckle-tracking technique, in the absence of wall-motion abnormalities, may represent a useful additional diagnostic finding in acute regional myocarditis.

Even though our sample size was small, we believe that the findings can be applied to patients with clinically suspected myocarditis. The 3D-speckle-tracking investigator was blinded to the results of the CMR. Nevertheless, further prospective studies on a larger collective with subdivision into different entities and follow-ups are required to confirm our results.

\section{Conclusions}

This is the first study investigating 3D-speckle-tracking echocardiography in patients with acute myocarditis. Global parameters, such as longitudinal strain, were significantly associated with the prevalence of myocarditis. 3D radial strain allows the diagnosis of regional wall mechanics, and, therefore, this new imaging modality could become a useful diagnostic tool in the diagnosis of myocarditis. A definitive diagnosis would still be based on clinical presentation and noninvasive diagnostic testing, such as CMR.

\section{Authors' contributions}

PRG, SZ, AZ, UB, JK, CH and VT acquired the Echo data, AI analyzed the cardiac MRI data, PRG, SZ, $\mathrm{AZ}, \mathrm{CH}, \mathrm{GN}$ and VT analyzed the data and wrote the manuscript.

\section{Figures and legends}

Figure 1: Study design. 45 patients with suspected acute myocarditis were included in this observational study. Each patient underwent standardized two-dimensional transthoracic echocardiography and a 3D fullvolume data set of the ventricle was obtained by using commercially available software (TomTec Imaging Systems $\mathrm{GmbH}$ ). All patients received cardiac MRI-scans and myocarditis was diagnosed in 29 subjects. 16 patients without myocarditis according to the CMR scan served as controls.

Figure 2: Correlation of CMR findings with the regional changes detected in the 3D-speckletracking. Regional changes in myocardial texture and tissue edema, as diagnosed by CMR, were significantly associated with regional impairment of circumferential, longitudinal, and radial strain as well as regional 3D displacement and total 3D strain. The 3D diastolic strain index was not associated with pathological findings in the CMR. Green: significantly associated (p[?]0.05); red: no significant association (p>0.05).

Figure 3: Assessment of the association of global 3D- and 2D parameters with acute myocarditis. Receiver operating characteristic curves for 3D-global longitudinal strain (GLS), LVEF, and LVEDV. 
GLS out-performed the well-known 2D parameters associated with myocarditis, LVEF and LVEDV. Global torsion and twist were not associated with myocarditis in our collective.

Figure 4: Example of the significant correlation of 3D radial-strain parameters with CMR findings in a patient with myocarditis. In the short axis stack view (left box) of the whole left ventricle, late enhancement of the anterior and lateral areas was detected in CMR. White arrows indicate the myocardial damage (hyperenhancement areas) that involve the subepicardial layers. This was significantly correlated with impairment in the 3D radial strain (right box) within the same area. RV: right venricle, LV: left ventricle.

Tables

\begin{tabular}{|c|c|c|c|c|c|c|}
\hline & All $(\mathrm{n}=45)$ & $\begin{array}{l}\text { Myocarditis } \\
(\mathrm{n}=29)\end{array}$ & $\begin{array}{l}\text { Controls } \\
(\mathrm{n}=16)\end{array}$ & $P$ & $P$ & \\
\hline 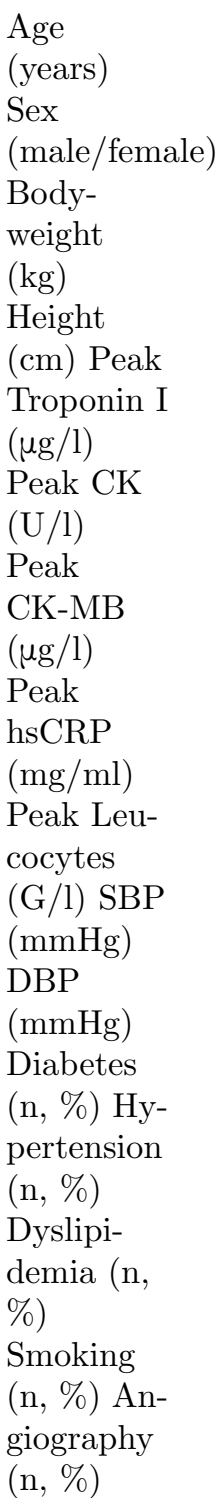 & $\begin{array}{l}43.9 \pm 16.3 \\
71 / 29 \% \\
(39 / 16) \\
85.7 \pm 17.5 \\
175.95 \pm 12.25 \\
1.38 \pm 3.51 \\
185.15 \pm 242.45 \\
20.5 \pm 35.8 \\
35.4 \pm 56.6 \\
9.72 \pm 3.94 \\
133.2 \pm 29.8 \\
79 \pm 11.73 \\
(5.5 \%) 13 \\
(23.6 \%) \\
20(36.4 \%) \\
11(20 \%) \\
38(69.1 \%)\end{array}$ & $\begin{array}{l}47.4 \pm 17.0 \\
72 / 28 \% \\
(21 / 8) \\
90.3 \pm 22.9 \\
175.1 \pm 11.9 \\
2.15 \pm 5.44 \\
218.4 \pm 331.7 \\
20.6 \pm 51.3 \\
39.5 \pm 49.6 \\
10.13 \pm 3.55 \\
131.2 \pm 24.5 \\
81.3 \pm 16.2 \\
3(10,3 \%) \\
8(27,6 \%) \\
10(34,5 \%) \\
6(20,7 \%) \\
23(79,3 \%)\end{array}$ & $\begin{array}{l}40.4 \pm 15.6 \\
63 / 37 \% \\
(10 / 6) \\
81.1 \pm 12.1 \\
176.8 \pm 12.6 \\
0.61 \pm 1.58 \\
151.9 \pm 153.2 \\
2.4 \pm 2.3 \\
31.3 \pm 63.6 \\
9.3 \pm 4.33 \\
135.1 \pm 35.0 \\
76.7 \pm 7.20 \\
(0 \%) 3 \\
(18,7 \%) 6 \\
(37,5 \%) 3 \\
(18,75 \%) 7 \\
(43,75 \%)\end{array}$ & $\begin{array}{l}40.4 \pm 15.6 \\
63 / 37 \% \\
(10 / 6) \\
81.1 \pm 12.1 \\
176.8 \pm 12.6 \\
0.61 \pm 1.58 \\
151.9 \pm 153.2 \\
2.4 \pm 2.3 \\
31.3 \pm 63.6 \\
9.3 \pm 4.33 \\
135.1 \pm 35.0 \\
76.7 \pm 7.20 \\
(0 \%) 3 \\
(18,7 \%) 6 \\
(37,5 \%) 3 \\
(18,75 \%) 7 \\
(43,75 \%)\end{array}$ & $\begin{array}{ll}0.18 & 0.37 \\
0.78 & 0.28 \\
0.45 & 0.17 \\
0.81 & 0.49 \\
0.71 & 0.41 \\
0.77 & 0,09 \\
0.24 & 0.44 \\
0.01 & \end{array}$ & $\begin{array}{ll}0.18 & 0.37 \\
0.78 & 0.28 \\
0.45 & 0.17 \\
0.81 & 0.49 \\
0.71 & 0.41 \\
0.77 & 0,09 \\
0.24 & 0.44 \\
0.01 & \end{array}$ \\
\hline
\end{tabular}


Table 1: Baseline clinical characteristics. Groups are divided with regard to the findings from CMR. Data are expressed as median or as percentage. CK: creatine kinase, CK-MB: muscle-brain type creatine kinase, hsCRP: high sensitivity C-reactive protein, SBP: systolic blood pressure, DBP: diastolic blood pressure

\begin{tabular}{lllll}
\hline & All $(\mathrm{n}=45)$ & $\begin{array}{l}\text { Myocarditis } \\
(\mathrm{n}=29)\end{array}$ & $\begin{array}{l}\text { Controls } \\
(\mathrm{n}=16)\end{array}$ & $P$ \\
\hline LVEF (\%) & $49.4 \pm 3.7$ & $44.4 \pm 3.2$ & $54.3 \pm 4.2$ & 0.030 .020 .08 \\
LVEDV (ml) & $135.6 \pm 14.4$ & $159.3 \pm 14.3$ & $111.8 \pm 14.4$ & 0.220 .560 .08 \\
LV length & $8.7 \pm 0.3$ & $8.9 \pm 0.2$ & $8.4 \pm 0.3$ & 0.030 .100 .004 \\
(cm) IVSd E & $1.2 \pm 0.07$ & $1.2 \pm 0.04$ & $1.1 \pm 0.09$ & 0.990 .080 .77 \\
wave (m/sec) & $0.86 \pm 0.06$ & $0.83 \pm 0.05$ & $0.88 \pm 0.07$ & 0.450 .930 .68 \\
A wave & $0.87 \pm 0.05$ & $0.55 \pm 0.04$ & $0.69 \pm 0.05$ & 0.88 \\
(m/sec) E/A & $1.48 \pm 0.16$ & $1.9 \pm 0.22$ & $1.05 \pm 0.09$ & \\
ratio Septal e & $0.26 \pm 0.06$ & $0.19 \pm 0.04$ & $0.33 \pm 0.08$ & \\
(cm/sec) & $8.83 \pm 1.33$ & $12.76 \pm 1.9$ & $4.9 \pm 0.76$ & $16.8 \pm 3.6$ \\
Septal E/e & $16.75 \pm 2.85$ & $16.7 \pm 2.1$ & $2.2 \pm 0.110$ & \\
ratio sPAP & $2.05 \pm 0.128$ & $1.89 \pm 0.118$ & $(62.5) 0(0) 2$ & \\
(mmHg) & $(62.2) 1(2.2) 7$ & $(62.1) 1(3.45)$ & $2.5) 1(6.25)$ & \\
TAPSE (cm) & $(15.6) 4(8.89)$ & $5(17.2) 3$ & $2.5)$ & \\
Primary & $5(11.1)$ & $(10.34) 3$ & & \\
ECG & & $(10.34)$ & & \\
findings & & & & \\
Normal n (\%) & & & & \\
ST-segment & & & & \\
elev. (\%) & & & & \\
T-wave & & & & \\
changes (\%) & & & & \\
RBBB (\%) & & & & \\
LBBB (\%) & & & &
\end{tabular}

Table 2: Conventional echocardiographic characteristics and primary ECG findings . LV, left ventricle; LVEF, left-ventricular ejection fraction; LVEDV, left-ventricular end-diastolic volume; IVSd, interventricular septum diameter; E, early; A, atrial; sPAP, systolic pulmonary arterial pressure; TAPSE, tricuspid annular plane systolic excursion; RBBB, right bundle branch block; LBBB, left bundle branch block. Data are expressed as the median or as a percentage.

\section{References}

1. Kindermann I, Barth C, Mahfoud F, Ukena C, Lenski M, Yilmaz A, et al. Update on myocarditis. Journal of the American College of Cardiology. 2012;59(9):779-92.

2. Pinamonti B, Alberti E, Cigalotto A, Dreas L, Salvi A, Silvestri F, et al. Echocardiographic findings in myocarditis. The American journal of cardiology. 1988;62(4):285-91.

3. Childs H, Friedrich MG. Cardiovascular magnetic resonance imaging in myocarditis. Progress in cardiovascular diseases. 2011;54(3):266-75.

4. Friedrich MG, Strohm O, Schulz-Menger J, Marciniak H, Luft FC, Dietz R. Contrast media-enhanced magnetic resonance imaging visualizes myocardial changes in the course of viral myocarditis. Circulation. 1998;97(18):1802-9.

5. Caforio AL, Pankuweit S, Arbustini E, Basso C, Gimeno-Blanes J, Felix SB, et al. Current state of 
knowledge on aetiology, diagnosis, management, and therapy of myocarditis: a position statement of the European Society of Cardiology Working Group on Myocardial and Pericardial Diseases. European heart journal. 2013;34(33):2636-48, 48a-48d.

6. Escher F, Kasner M, Kuhl U, Heymer J, Wilkenshoff U, Tschope C, et al. New echocardiographic findings correlate with intramyocardial inflammation in endomyocardial biopsies of patients with acute myocarditis and inflammatory cardiomyopathy. Mediators of inflammation. 2013;2013:875420.

7. Di Bella G, Coglitore S, Zimbalatti C, Minutoli F, Zito C, Patane S, et al. Strain Doppler echocardiography can identify longitudinal myocardial dysfunction derived from edema in acute myocarditis. Int J Cardiol. 2008;126(2):279-80.

8. Leitman M, Vered Z, Tyomkin V, Macogon B, Moravsky G, Peleg E, et al. Speckle tracking imaging in inflammatory heart diseases. The international journal of cardiovascular imaging. 2018;34(5):787-92.

9. Di Bella G, Gaeta M, Pingitore A, Oreto G, Zito C, Minutoli F, et al. Myocardial deformation in acute myocarditis with normal left ventricular wall motion-a cardiac magnetic resonance and 2-dimensional strain echocardiographic study. Circulation journal : official journal of the Japanese Circulation Society. 2010;74(6):1205-13.

10. Kostakou PM, Kostopoulos VS, Tryfou ES, Giannaris VD, Rodis IE, Olympios CD, et al. Subclinical left ventricular dysfunction and correlation with regional strain analysis in myocarditis with normal ejection fraction. A new diagnostic criterion. Int J Cardiol. 2018;259:116-21.

11. Hsiao JF, Koshino Y, Bonnichsen CR, Yu Y, Miller FA, Jr., Pellikka PA, et al. Speckle tracking echocardiography in acute myocarditis. The international journal of cardiovascular imaging. 2013;29(2):27584.

12. Lang RM, Badano LP, Mor-Avi V, Afilalo J, Armstrong A, Ernande L, et al. Recommendations for cardiac chamber quantification by echocardiography in adults: an update from the American Society of Echocardiography and the European Association of Cardiovascular Imaging. European heart journal cardiovascular Imaging. 2015;16(3):233-70.

13. Quinones MA, Otto CM, Stoddard M, Waggoner A, Zoghbi WA, Doppler Quantification Task Force of the N, et al. Recommendations for quantification of Doppler echocardiography: a report from the Doppler Quantification Task Force of the Nomenclature and Standards Committee of the American Society of Echocardiography. Journal of the American Society of Echocardiography : official publication of the American Society of Echocardiography. 2002;15(2):167-84.

14. Germain P, El Ghannudi S, Jeung MY, Ohlmann P, Epailly E, Roy C, et al. Native T1 mapping of the heart - a pictorial review. Clinical Medicine Insights Cardiology. 2014;8(Suppl 4):1-11.

15. Luetkens JA, Doerner J, Schild H, Naehle CP. Diagnostic value of quantitative CMR in patients suspected of having myocarditis: a question of timing. JACC Cardiovascular imaging. 2015;8(1):109-10.

16. Muraru D, Niero A, Rodriguez-Zanella H, Cherata D, Badano L. Three-dimensional speckle-tracking echocardiography: benefits and limitations of integrating myocardial mechanics with three-dimensional imaging. Cardiovascular diagnosis and therapy. 2018;8(1):101-17.

17. Amzulescu MS, De Craene M, Langet H, Pasquet A, Vancraeynest D, Pouleur AC, et al. Myocardial strain imaging: review of general principles, validation, and sources of discrepancies. European heart journal cardiovascular Imaging. 2019;20(6):605-19.

18. Felker GM, Boehmer JP, Hruban RH, Hutchins GM, Kasper EK, Baughman KL, et al. Echocardiographic findings in fulminant and acute myocarditis. Journal of the American College of Cardiology. 2000;36(1):227-32. 
19. Nieminen MS, Heikkila J, Karjalainen J. Echocardiography in acute infectious myocarditis: relation to clinical and electrocardiographic findings. The American journal of cardiology. 1984;53(9):1331-7.

20. James KB, Lee K, Thomas JD, Hobbs RE, Rincon G, Bott-Silverman C, et al. Left ventricular diastolic dysfunction in lymphocytic myocarditis as assessed by Doppler echocardiography. The American journal of cardiology. 1994;73(4):282-5.

21. Lieback E, Hardouin I, Meyer R, Bellach J, Hetzer R. Clinical value of echocardiographic tissue characterization in the diagnosis of myocarditis. European heart journal. 1996;17(1):135-42.

22. Tops LF, Delgado V, Marsan NA, Bax JJ. Myocardial strain to detect subtle left ventricular systolic dysfunction. European journal of heart failure. 2017;19(3):307-13.

23. Jeung MY, Germain P, Croisille P, El ghannudi S, Roy C, Gangi A. Myocardial tagging with MR imaging: overview of normal and pathologic findings. Radiographics : a review publication of the Radiological Society of North America, Inc. 2012;32(5):1381-98.

24. Kalam K, Otahal P, Marwick TH. Prognostic implications of global LV dysfunction: a systematic review and meta-analysis of global longitudinal strain and ejection fraction. Heart. 2014;100(21):1673-80.

25. Biering-Sorensen T, Biering-Sorensen SR, Olsen FJ, Sengelov M, Jorgensen PG, Mogelvang R, et al. Global Longitudinal Strain by Echocardiography Predicts Long-Term Risk of Cardiovascular Morbidity and Mortality in a Low-Risk General Population: The Copenhagen City Heart Study. Circulation Cardiovascular imaging. 2017;10(3), e005521.

26. Diao KY, Yang ZG, Ma M, He Y, Zhao Q, Liu X, et al. The Diagnostic Value of Global Longitudinal Strain (GLS) on Myocardial Infarction Size by Echocardiography: A Systematic Review and Meta-analysis. Scientific reports. 2017;7(1):10082.

27. Ng ACT, Prihadi EA, Antoni ML, Bertini M, Ewe SH, Ajmone Marsan N, et al. Left ventricular global longitudinal strain is predictive of all-cause mortality independent of aortic stenosis severity and ejection fraction. European heart journal cardiovascular Imaging. 2018;19(8):859-67.

28. Tower-Rader A, Mohananey D, To A, Lever HM, Popovic ZB, Desai MY. Prognostic Value of Global Longitudinal Strain in Hypertrophic Cardiomyopathy: A Systematic Review of Existing Literature. JACC Cardiovascular imaging. 2018, pii: S1936-878X(18)30671-5.

29. Ha SJ, Woo JS, Kwon SH, Oh CH, Kim KS, Bae JH, et al. Acute regional myocarditis with normal ventricular wall motion diagnosed by two-dimensional speckle tracking imaging. The Korean journal of internal medicine. 2013;28(6):732-5.

\section{Hosted file}

Abb_Myo_new_15_02.pdf available at https://authorea.com/users/396399/articles/509590-3dspeckle-tracking-echocardiography-correlates-with-cmr-diagnosis-of-acute-myocarditis 